\title{
A review of biomechanical studies for mandibular angle fracture internal fixation.
}

\author{
Fernando P.S. Guastaldi ${ }^{1}{ }^{*}$, Joao L.G.C. Monteiro ${ }^{1}$, Toru Takusagawa ${ }^{1}$, Antonio Carlos Guastaldi ${ }^{2}$ \\ ${ }^{1}$ Skeletal Biology Research Center, Department of Oral and Maxillofacial Surgery, Massachusetts General Hospital, Harvard School of Dental
} Medicine, Boston, MA, USA.

${ }^{2}$ Biomaterials Group, Department of Physical Chemistry, São Paulo State University (Unesp), Institute of Chemistry, Araraquara, SP, Brazil.

*Corresponding Author: Fernando P.S. Guastaldi, Department of Oral and Maxillofacial Surgery, Massachusetts General Hospital, Harvard School of Dental Medicine, Boston, MA, USA.

Received Date: August 23 2021; Accepted Date: September 10 2021; Published Date: September 14, 2021

Citation: F.P.S. Guastaldi, J.L.G.C. Monteiro, T. Takusagawa, A.C. Guastaldi. A review of biomechanical studies for mandibular angle fracture internal fixation, J Dentistry and Oral Maxillofacial Surgery.4(1) DOI: 10.31579/2643-6612/020

Copyright: () 2021 :Fernando P.S. Guastaldi, this is an open-access article distributed under the terms of the Creative Commons Attribution License, which permits unrestricted use, distribution, and reproduction in any medium, provided the original author and source are credited.

\begin{abstract}
Mandible fractures correspond to $19-40 \%$ of all facial fractures. Among all mandible fractures, $12-30 \%$ are fractures of the mandibular angle. These fractures are mainly caused by sports activities, interpersonal violence and car accidents. The presence of the third molar and the thin transverse bone area seem to be responsible for the frequent involvement of the mandibular angle in facial fractures. Before the advent of antibiotics, a high frequency of infection was always associated after an open reduction in mandibular angle fractures. Wired osteosynthesis and maxillomandibular fixation (MMF) were traditional methods for fixing mandibular angle fractures. The limitation of both methods has influenced the development of new approaches for the treatment of mandibular angle fractures. Currently, osteosynthesis of mandibular angle fractures with plates and screws has become an effective treatment option. Several forms are described in the literature as: fixation of bone segments with a miniplate on the upper edge of the mandible, fixation with two miniplates, lag screw or by a single rigid plate on the lower edge of the mandible. The purpose of this study was to summarize the main characteristics of biomechanical studies such as the type of mandible source used, the plating techniques employed, the plate material, and the loading protocols used to evaluate the stability of the fixation methods. The Medline (PubMed) database was searched combining relevant terms and pertinent articles in English were included. Articles had to meet the following inclusion criteria: be in vitro biomechanical studies evaluating fixation methods for mandibular angle fractures. A total of 27 articles fulfilled the inclusion criteria. Synthetic mandibles $(n=12)$, animal mandibles $(n=9)$, and human cadaveric mandibles $(n=5)$ were used as the sample source to perform the biomechanical analysis. One article used both synthetic and human cadaveric mandibles. Also, a variety of fixation techniques was described such as mini-plates, lag-screws, reconstruction plates, and three-dimensional plates. The materials of the bone plates used were: stainless steel, commercially pure titanium, titanium alloy or bioresorbable. However, there was inconsistency in reporting the materials and not all studies clearly stated the material of the bone plates. For the biomechanical analysis, there was a high variation among all studies regarding the loading protocols used. The same side of fixation, the anterior part of the mandible (central incisors) or the contralateral side were reported as the dentate regions in which the force was applied. For more than 2 decades, in vitro biomechanical studies have been used to help researchers and clinicians in the field of Oral and Maxillofacial Surgery to properly evaluate and compare the different devices and techniques available for the treatment of mandibular angle fractures. Also, biomechanical studies are important to answer questions on fatigue performance and fracture strength on the gross level.
\end{abstract}

Keywords: Mandibular angle fractures; internal fixation; biomechanics, mechanical testing.

\section{Introduction}

The mandible is the largest, strongest and the only movable stress bearing bone of the face (Wong et al., 2011). However, position, prominence, anatomic configuration, mobility, and less bone support make the mandibular bone fractures occurring twice as frequently as fractures of the midface (de Matos et al., 2010). The distribution of fracture sites seems to be influenced by the cause of the injury, which in turn is influenced by geographic locations, local behavior, and socioeconomic trends (Erdmann et al., 2008; Bormann et al., 2009). Mostly, these injuries are related to a traumatic incidence including traffic accident, interpersonal violence, falls, and sport injuries (Zachariades et al., 2006; Jain et al., 2009).Mandible fractures correspond to $19-40 \%$ of all facial fractures. Of these, 12-30\% account for fractures of the mandibular angle (Allareddy et al., 2011) (Figure 1). 


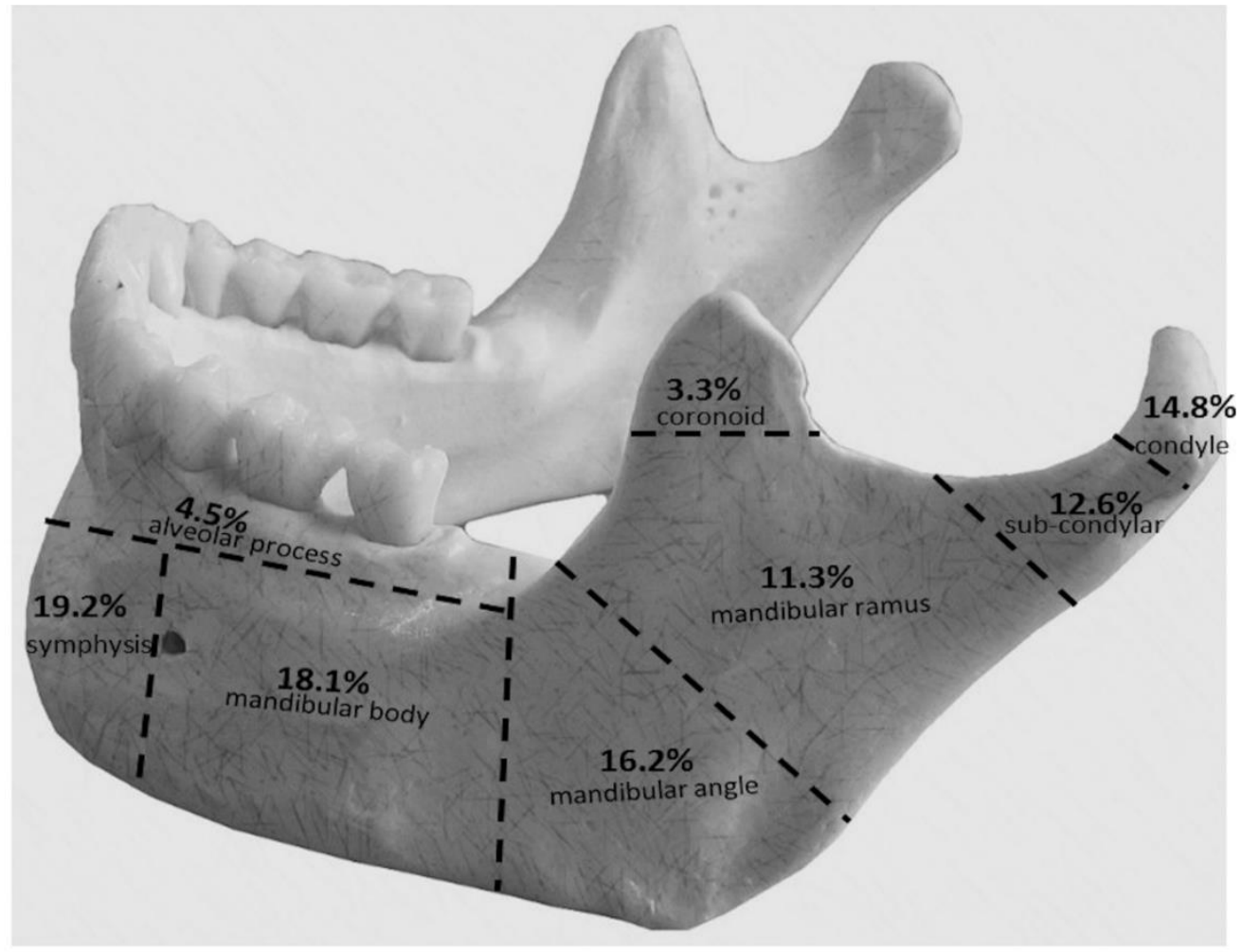

Figure 1: Prevalence of fractures according to regions of the mandible (data extracted from Afrooz et al., 2015).

This can be attributed to its relatively thin cross-sectional area and its location near the third molar tooth socket (Fonseca, 2005). Due to these anatomical features compared to other mandibular body parts the frequency of occurrence increases.

The management of mandibular angle fractures has traditionally been associated with a high postoperative complication rate (Iizuka et al., 1991; Schmelzeisen et al., 1992; Gabrielli et al., 2003). Physiologic, anatomic, and social factors may contribute to these complications. The mandibular angle is subject to muscle forces related to the muscles of mastication and suprahyoid musculature resulting in unstable rotation of the proximal and distal fracture segments (Schierle et al., 1997).

Wired osteosynthesis and maxillomandibular fixation (MMF) were traditional methods for fixing mandibular angle fractures. The limitation of both methods has influenced the development of new approaches for the treatment of mandibular angle fractures. Many methods of osteosynthesis for mandibular angle fractures have been described, including fixation of fragments with compression plates, reconstruction plates, locking reconstruction plates, lag screws, miniplates, bioresorbable plates, and three-dimensional miniplates (Shetty \& Caputo, 1992; Ellis \& Sinn, 1993; Ellis, 1993, 1999).

As new materials and techniques are developed to be used in the treatment of mandibular angle fractures, preclinical studies represent the first step towards clinical application. For more than 2 decades, in vitro biomechanical studies have been used to help researchers and clinicians in the field of Oral and Maxillofacial Surgery to properly evaluate and compare the different devices and techniques available for the treatment of mandibular angle fractures. Also, biomechanical studies are important to answer questions on fatigue performance and fracture strength on the gross level.

Therefore, the purpose of this study was to summarize the main characteristics of biomechanical studies such as the type of mandible source used, the plating techniques employed, the plate material, and the loading protocols used to evaluate the stability of the fixation methods.

\section{Methods}

A computer database search was performed, using Medline (PubMed) without data restriction. The following search criteria was used: (("mandible"[MeSH Terms] OR "mandible"[All Fields]) OR ("mandible"[MeSH Terms] OR "mandible"[All Fields] OR "mandibular"[All Fields])) AND (("fractures, bone"[MeSH Terms] OR ("fractures"[All Fields] AND "bone"[All Fields]) OR "bone fractures"[All Fields] OR "fracture"[All Fields]) OR ("injuries"[Subheading] OR "injuries"[All Fields] OR "trauma"[All Fields] OR "wounds and injuries"[MeSH Terms] OR ("wounds"[All Fields] AND "injuries"[All Fields]) OR "wounds and injuries"[All Fields]) OR ("wounds and injuries"[MeSH Terms] OR ("wounds"[All Fields] AND "injuries"[All Fields]) OR "wounds and injuries"[All Fields] OR "injury"[All Fields])) AND (angle[All Fields] OR angulus[All Fields]) AND (biomechanical[All Fields] OR ("in vitro techniques"[MeSH Terms] OR ("vitro"[All Fields] AND "techniques"[All Fields]) OR "in vitro techniques"[All Fields] OR "vitro"[All Fields] OR "in vitro"[All Fields])).

Terms were limited to studies that were published in the English language. Studies were included if models were used for looking at the biomechanics of any method of mandibular angle fracture fixation either alone or in combination with clinical studies. Studies were excluded if the full text wasn't available, if they reported on models used any other site of mandibular fracture fixation, bone graft for mandibular reconstruction, orthognathic surgery, distraction osteogenesis, dental implants and dental prosthesis. Any papers reporting only clinical outcomes were also excluded.

Articles that met the inclusion criteria based on their abstract information were selected. Articles were also obtained when there was not enough information in the abstracts or if a citation had no abstract. 
The following data was extracted from each article: author(s), year of publication, title, sample source (human, animal or synthetic), plating technique and plate material, and load-bearing area (for the biomechanical analysis). These data were summarized in figures and tables and descriptive statistic was provided when appropriate.

\section{Results}

The search (performed on March 11th, 2020) yielded a total of 134 titles. After reading the titles and abstracts, a total of 27 articles were selected for reading the full text and for data extraction (Table 1).

\begin{tabular}{|c|c|c|}
\hline Author(s) & Year & Title \\
\hline Said et al. & 2019 & The effect of the mandibular plane angle on fracture line stability: An ex vivo experimental study \\
\hline Cankaya et al. & 2018 & $\begin{array}{l}\text { Effects of polymethylmethacrylate on the stability of screw fixation in mandibular angle fractures: A study on } \\
\text { sheep mandibles }\end{array}$ \\
\hline Sittitavornwong et al. & 2018 & $\begin{array}{l}\text { Integrity of a Single Superior Border Plate Repair in Mandibular Angle Fracture: A Novel Cadaveric Human } \\
\text { Mandible Model }\end{array}$ \\
\hline Cural et al. & 2018 & $\begin{array}{l}\text { Comparison of Mechanical Stabilization of the Mandibular Angulus Fracture Fixation, With Titanium Plates } \\
\text { and Screws, Resorbable Plates and Screws, and Bone Adhesives }\end{array}$ \\
\hline Zimmermann et al. & 2017 & $\begin{array}{l}\text { Biomechanical comparison of a multidirectional locking plate and conventional plates for the osteosynthesis of } \\
\text { mandibular angle fractures: A preliminary study }\end{array}$ \\
\hline Wallner et al. & 2017 & $\begin{array}{l}\text { Osteosynthesis using cannulated headless Herbert screws in mandibular angle fracture treatment: A new } \\
\text { approach? }\end{array}$ \\
\hline Pereira-Filho et al. & 2016 & Evaluation of Three Different Osteosynthesis Methods for Mandibular Angle Fractures: Vertical Load Test \\
\hline Medeiros et al. & 2016 & $\begin{array}{l}\text { In Vitro Mechanical Analysis of Different Techniques of Internal Fixation of Combined Mandibular Angle and } \\
\text { Body Fractures }\end{array}$ \\
\hline Lieger et al. & 2015 & Biomechanical evaluation of different angle-stable locking plate systems for mandibular surgery \\
\hline $\begin{array}{l}\text { Muñante-Cardenas } \\
\text { and Passeri }\end{array}$ & 2015 & Biomechanical Comparison of Four Mandibular Angle Fracture Fixation Techniques \\
\hline Jafarian et al. & 2015 & $\begin{array}{l}\text { Assessment of compression and strength of divergent screws mounted on miniplates for fixation of } \\
\text { mandibular fractures: an in vitro experimental study }\end{array}$ \\
\hline Guastaldi et al. & 2014 & $\begin{array}{l}\text { Biomechanical study in polyurethane mandibles of different metal plates and internal fixation techniques, } \\
\text { employed in mandibular angle fractures }\end{array}$ \\
\hline Suer et al. & 2014 & Biomechanical evaluation of a new design titanium miniplate for the treatment of mandibular angle fractures \\
\hline Negreiros et al. & 2014 & Comparison of compressive strength between three different plates for mandibular angle fractures fixation \\
\hline Trivellato et al. & 2014 & $\begin{array}{l}\text { In vitro evaluation of the resistance of a } 2.0-\mathrm{mm} \text { titanium fixation system in the sectioned angle without } \\
\text { continuity of the inferior border of the mandible. }\end{array}$ \\
\hline Pektas et al. & 2012 & $\begin{array}{l}\text { Effects of different mandibular fracture patterns on the stability of miniplate screw fixation in angle } \\
\text { mandibular fractures }\end{array}$ \\
\hline Ribeiro-Jr et al. & 2010 & $\begin{array}{l}\text { In vitro evaluation of conventional and locking miniplate/screw systems for the treatment of mandibular angle } \\
\text { fractures }\end{array}$ \\
\hline Kalfarentzos et al. & 2009 & $\begin{array}{l}\text { Biomechanical evaluation of plating techniques for fixing mandibular angle fractures: the introduction of a new } \\
\text { 3D plate approach }\end{array}$ \\
\hline Turgut et al. & 2008 & $\begin{array}{l}\text { Biomechanical comparison of a new technique of mandibular angle fractures: biplanar and bicortical superior } \\
\text { proximal } 3 \text { holes and bicortical inferior plate fixation }\end{array}$ \\
\hline
\end{tabular}




\begin{tabular}{|l|l|l|}
\hline Alkan et al. & 2007 & Biomechanical comparison of different plating techniques in repair of mandibular angle fractures \\
\hline Haug et al. & 2002 & Does plate adaptation affect stability? A biomechanical comparison of locking and nonlocking plates \\
\hline Haug et al. & 2001 & A biomechanical evaluation of mandibular angle fracture plating techniques \\
\hline Wittenberg et al. & 1997 & Biomechanical evaluation of new fixation devices for mandibular angle fractures \\
\hline Haug et al. & 1996 & A comparison of mandibular angle fracture plating techniques \\
\hline Shetty et al. & 1995 & $\begin{array}{l}\text { Fracture line stability as a function of the internal fixation system: an in vitro comparison using a mandibular } \\
\text { angle fracture model }\end{array}$ \\
\hline Choi et al. & 1995 & Stability testing of a two miniplate fixation technique for mandibular angle fractures. An in vitro study \\
\hline & 1992 & Biomechanical validation of the solitary lag screw technique for reducing mandibular angle fractures \\
\hline Shetty \& Caputo & & \\
\hline
\end{tabular}

\section{Table 1: Reference of the selected papers.}

Nine studies were performed in mandibles derived from animals. In these studies, sheep mandibles were used. Four studies used mandibles derived from human donors, and 11 studies used synthetic mandibles, mostly composed of polyurethane. One study performed analysis in both human cadaveric and synthetic mandibles (Figure 2).

\begin{tabular}{|c|c|c|c|}
\hline Author(s)/Year & Sample source & Plating technique & Load-bearing area \\
\hline Said et al. 2019 & Animal (sheep) & $\begin{array}{l}\text { Single four-hole titanium } 2.0 \text { miniplate at the } \\
\text { superior border fixed with } 5.0 \text { m-mm-long } \\
\text { screw. This fixation was applied to mandibles } \\
\text { in which different mandibular plane angles } \\
\text { were created }\end{array}$ & $\begin{array}{l}\text { Vertical loading at the molar teeth (side of } \\
\text { fixation) }\end{array}$ \\
\hline $\begin{array}{l}\text { Cankaya et al. } \\
2018\end{array}$ & Animal (sheep) & $\begin{array}{l}\text { Two-techniques: } \\
\text { Four-hole non-compression titanium } \\
\text { miniplate }(1 \mathrm{~mm} \text { thick) at the center of } \\
\text { fracture line fixed with screws }(2.0 \mathrm{~mm} \text { in } \\
\text { diameter and } 11 \mathrm{~mm} \text { long) and PMMA } \\
\text { applied to the screw tracts } \\
\text { Four-hole non-compression titanium } \\
\text { miniplate }(1 \mathrm{~mm} \text { thick) at the center of } \\
\text { fracture line fixed with screws ( } 2.0 \mathrm{~mm} \text { in } \\
\text { diameter and } 11 \mathrm{~mm} \text { long) } \\
\text { All the plates were from Medplates (Istanbul, } \\
\text { Turkey) }\end{array}$ & $\begin{array}{l}\text { Vertical loading at the molar teeth (side of } \\
\text { fixation) }\end{array}$ \\
\hline $\begin{array}{l}\text { Sittitavornwong } \\
\text { et al. } 2018\end{array}$ & Cadaveric & $\begin{array}{l}\text { Single plate at the upper border of mandibular } \\
\text { body (conventional curved titanium } 2.06 \text {-hole } \\
\text { noncompression miniplate }\end{array}$ & $\begin{array}{l}\text { Vertical loading at the first molar (side of } \\
\text { fixation) }\end{array}$ \\
\hline $\begin{array}{l}\text { Cural et al. } \\
2018\end{array}$ & Animal (sheep) & $\begin{array}{l}\text { Single plate at the external oblique ridge } \\
\text { with } 3 \text { techniques: } \\
\text { Four-hole titanium } 2.0 \mathrm{~mm} \text { plate (Champy } \\
\text { Module, Osteosynthese } 2.0 \text { mini, KLS Martin, } \\
\text { Umkirch, Germany) } \\
\text { Five-hole resorbable PDLLA plate (Resorb-x } \\
\text { plates \& meshes, KLS Martin) } \\
\text { Five-hole resorbable poly-DL-lactic acid } \\
\text { (PDLLA) plate (Resorb-x plates \& meshes, }\end{array}$ & Unclear \\
\hline
\end{tabular}




\begin{tabular}{|c|c|c|c|}
\hline & & $\begin{array}{l}\text { KLS Martin). No screws were used and an } \\
\text { adhesive (2-OSA + BLSA) was applied to the } \\
\text { plate and fragments were held together for } \\
120 \text { seconds until polymerization } \\
\text { One group fixated by applying an adhesive } \\
\text { only }\end{array}$ & \\
\hline $\begin{array}{l}\text { Zimmermann et } \\
\text { al. } 2017\end{array}$ & $\begin{array}{l}\text { Synthetic } \\
\text { (polyurethane) }\end{array}$ & $\begin{array}{l}\text { Four techniques: } \\
\text { Four-hole locking plate at the external oblique } \\
\text { ridge (Tifix }{ }^{\circledR} \text { Mandible } 2.0 \text {, straight plate, } \\
\text { Litos, Ahrens-burg, Germany) } \\
\text { Five-hole non-locking plate (Maxillofazial } \\
\text { 2.0, Litos, Ahrensburg, Germany) at the } \\
\text { external oblique ridge + two-hole plate at the } \\
\text { caudal edge of the mandible. }^{\text {a }} \\
\text { Four-hole plate (Medartis }{ }^{\circledR}, \text { Basel, } \\
\text { Switzerland) at the external oblique ridge + } \\
\text { three-hole plate at the caudal edge of the } \\
\text { mandible. } \\
\text { Six-hole non-locking plate at the external } \\
\text { oblique ridge (Medartis }{ }^{\circledR}, \text { Basel, Switzerland; } \\
\text { mandibular plate prebent } 70, \text { Modus } 2.0 \text { ) }\end{array}$ & $\begin{array}{l}\text { Vertical loading through a steel panel placed at } \\
\text { the lower incisors }\end{array}$ \\
\hline $\begin{array}{l}\text { Wallner et al. } \\
2017\end{array}$ & Cadaveric & $\begin{array}{l}\text { Two techniques (bilateral angle fractures, } \\
\text { one technique per side): } \\
\text { Conventional titanium } 2.0 \text { four-hole } \\
\text { mandibular angle locking plates (TriLock } 2.0 \text {, } \\
\text { Med Artis Modus) with four monocortical, } \\
\text { titanium fixation locking screws (TriLock } \\
\text { fixation screw, outer thread diameter: } 2.0 \mathrm{~mm} \text {, } \\
\text { length: } 8 \mathrm{~mm} \text {, MedArtis Modus) } \\
\text { Cannulated, headless, bicortical, titanium type } \\
2 \text { Herbert bone screws (HBS } 2 \text {; mini, short } \\
\text { thread, proximal outer thread diameter: } 3.2 \\
\text { mm, distal outer thread diameter: } 2.5 \mathrm{~mm} \text {, } \\
\text { threadless middle diameter: } 1.7 \text { mm, proximal } \\
\text { thread gradient: } 0.75 \mathrm{~mm} \text {, distal thread } \\
\text { gradient: } 1.0 \mathrm{~mm} \text {, KLS Martin) }\end{array}$ & $\begin{array}{l}\text { Vertical loading between the first and second } \\
\text { molar (side of fixation) }\end{array}$ \\
\hline $\begin{array}{l}\text { Pereira-Filho et } \\
\text { al. } 2016\end{array}$ & $\begin{array}{l}\text { Synthetic } \\
\text { (polyurethane) }\end{array}$ & $\begin{array}{l}\text { Three techniques: } \\
\text { Four-hole } 2.0 \mathrm{~mm} \text { system straight plate at the } \\
\text { external oblique ridge } \\
\text { Eight-hole } 2.0 \mathrm{~mm} \text { system curved grid plate } \\
\text { applied to the neutral zone of the mandible } \\
\text { Four-hole } 2.0 \mathrm{~mm} \text { system grid plate applied to } \\
\text { the neutral zone of the mandible } \\
\text { All plates were obtained from Engimplan (Rio } \\
\text { Claro, Brazil) }\end{array}$ & $\begin{array}{l}\text { Vertical loading between the canine and first } \\
\text { bicuspid (side of fixation) }\end{array}$ \\
\hline $\begin{array}{l}\text { Muñante- } \\
\text { Cardenas and } \\
\text { Passeri }\end{array}$ & Synthetic & $\begin{array}{l}\text { Four techniques: } \\
\text { Four-hole miniplate }(1 \mathrm{~mm} \times 23.5 \mathrm{~mm} \times 5.5 \\
\mathrm{mm}) \text { at the external oblique ridge } \\
\text { Two four-hole miniplates ( } 1 \mathrm{~mm} \times 23.5 \mathrm{~mm} \times \\
5.5 \mathrm{~mm}) \text { in parallel on the fracture line } \\
\text { 3D four-hole plate }(1 \mathrm{~mm} \times 20 \mathrm{~mm} \times 11.5 \\
\mathrm{mm}) \\
\text { 3D eight-hole plate }(0.8 \mathrm{~mm} \times 31.5 \mathrm{~mm} \times 10 \\
\mathrm{mm}) \\
\text { All plates were obtained from Engimplan (Rio } \\
\text { Claro, Brazil) }\end{array}$ & $\begin{array}{l}\text { Vertical load the first molar or in the central } \\
\text { incisor (side of fixation) }\end{array}$ \\
\hline $\begin{array}{l}\text { Jafarian et al. } \\
2015\end{array}$ & Animal (sheep) & $\begin{array}{l}\text { Two techniques (bilateral angle fractures, } \\
\text { one technique per side): } \\
\text { Four-hole titanium miniplate without a bar } \\
\text { (system } 2.0 \text {, General-Implants } \mathrm{GmbH} \\
\text { Deutschland) with four-hole } 4 \text { x } 7 \mathrm{~mm} \text { self- } \\
\text { tapping crosshead screws } 2 \mathrm{~mm} \text { in diameter } \\
\text { Four-hole titanium miniplate without a bar } \\
\text { (system } 2.0, \text { General-Implants GmbH }\end{array}$ & Vertical load the first molar (side of fixation) \\
\hline
\end{tabular}




\begin{tabular}{|c|c|c|c|}
\hline & & $\begin{array}{l}\text { Deutschland) with four-hole } 4 \times 7 \mathrm{~mm} \text { self- } \\
\text { tapping crosshead screws } 2 \mathrm{~mm} \text { in diameter; } \\
\text { however, the drilling and insertion of the } \\
\text { screws were } 45^{\circ} \text { divergent }\end{array}$ & \\
\hline $\begin{array}{l}\text { Guastaldi et al. } \\
2014\end{array}$ & $\begin{array}{l}\text { Synthetic } \\
\text { (polyurethane) }\end{array}$ & $\begin{array}{l}\text { Four techniques: } \\
\text { Four-hole } 2.0 \mathrm{~mm} \text { titanium plate fixed with } 4 \\
\text { monocortical screws } 6 \mathrm{~mm} \text { long in the tension } \\
\text { zone of the mandible } \\
\text { Four-hole } 2.0 \mathrm{~mm} \text { titanium plate fixed with } 4 \\
\text { monocortical screws } 6 \mathrm{~mm} \text { long in the tension } \\
\text { zone of the mandible }+ \text { four-hole } 2.0 \mathrm{~mm} \\
\text { titanium plate fixed with } 12 \mathrm{~mm} \text { long screws } \\
\text { in the compression zone } \\
\text { Four-hole } 2.0 \mathrm{~mm} \text { titanium-molybdenum plate } \\
\text { fixed with } 4 \text { monocortical screws } 6 \mathrm{~mm} \text { long } \\
\text { in the tension zone of the mandible } \\
\text { Four-hole } 2.0 \text { mm titanium plate fixed with } 4 \\
\text { monocortical screws } 6 \text { mm long in the tension } \\
\text { zone of the mandible }+ \text { four-hole } 2.0 \mathrm{~mm} \\
\text { titanium-molybdenum plate fixed with } 12 \mathrm{~mm} \\
\text { long screws in the compression zone } \\
\text { All plates were obtained from Engimplan (Rio } \\
\text { Claro, Brazil) }\end{array}$ & Vertical load at the first molar (side of fixation) \\
\hline Suer et al. 2014 & Animal (sheep) & $\begin{array}{l}\text { Two techniques: } \\
\text { Titanium non-compression miniplate with a } \\
\text { new-design fixed with } 2.0 \text { x } 6.0 \mathrm{~mm} \\
\text { monocortical titanium screws at the external } \\
\text { oblique ridge } \\
\text { Titanium six-hole straight non-compression } \\
\text { miniplate fixed with } 2.0 \text { x } 6.0 \mathrm{~mm} \\
\text { monocortical titanium screws at the external } \\
\text { oblique ridge } \\
\text { All plates were obtained from Trimed } \\
\text { Titanium Implant Systems; (Trimed, Ankara, } \\
\text { Turkey) }\end{array}$ & $\begin{array}{l}\text { Vertical, lateral and tensile forces in a testing } \\
\text { machine which simulates the masticatory muscles }\end{array}$ \\
\hline $\begin{array}{l}\text { Negreiros et al. } \\
2014\end{array}$ & $\begin{array}{l}\text { Synthetic } \\
\text { (polyurethane) }\end{array}$ & $\begin{array}{l}\text { Three techniques: } \\
\text { Four-hole } 2.0 \text { titanium plate }(1 \mathrm{~mm} \times 25 \mathrm{~mm} \\
\times 5.5 \mathrm{~mm}) \text { fixed with } 6.0 \text { screws at the } \\
\text { external oblique ridge } \\
\text { Four-hole } 2.0 \text { titanium grid plate }(1 \mathrm{~mm} \times 20 \\
\mathrm{mm} \times 11.5 \mathrm{~mm}) \text { fixed with } 6.0 \text { screws at the } \\
\text { intermediary portion of the mandible } \\
\text { Four-hole } 2.0 \text { titanium grid plate }(1 \mathrm{~mm} \times 20 \\
\mathrm{mm} \times 11.5 \mathrm{~mm}) \text { with middle reinforcement } \\
\text { fixed with } 6.0 \text { screws at the intermediary } \\
\text { portion of the mandible }\end{array}$ & $\begin{array}{l}\text { Vertical load at the canines and first premolar } \\
\text { (side of fixation) }\end{array}$ \\
\hline $\begin{array}{l}\text { Trivellato et al. } \\
2014\end{array}$ & $\begin{array}{l}\text { Synthetic } \\
\text { (polyurethane) }\end{array}$ & $\begin{array}{l}\text { One } 2.0 \text { titanium four-hole miniplate }(1.0 \mathrm{~mm} \\
\text { thick, } 23.5 \mathrm{~mm} \text { long, with } 4.5 \mathrm{~mm} \text { between } \\
\text { the center of the holes) fixed with } 6.0 \mathrm{~mm} \\
\text { long self-tapping screws (fracture patterns } \\
\text { with or without continuity of the mandible } \\
\text { were used) }\end{array}$ & $\begin{array}{l}\text { Vertical load the first molar (side of fixation and } \\
\text { contralateral side) and between the central } \\
\text { incisors }\end{array}$ \\
\hline $\begin{array}{l}\text { Pektas et al. } \\
2012\end{array}$ & Animal (sheep) & $\begin{array}{l}\text { One four-hole straight titanium miniplate } \\
\text { fixed with } 2.0 \mathrm{~mm} \times 7 \mathrm{~mm} \text { screws at the } \\
\text { external oblique ridge (fracture patterns with } \\
\text { or without continuity of the mandible were } \\
\text { used) }\end{array}$ & Vertical load at the posterior mandible \\
\hline
\end{tabular}




\begin{tabular}{|c|c|c|c|}
\hline $\begin{array}{l}\text { Ribeiro-Jr et al. } \\
2010\end{array}$ & $\begin{array}{l}\text { Synthetic } \\
\text { (polyurethane } \\
\text { hemimandibles) }\end{array}$ & $\begin{array}{l}2.0 \mathrm{~mm} \text { miniplates and } 2.0 \mathrm{~mm} \text { diameter } \mathrm{x} \\
6 \mathrm{~mm} \text { long monocortical screws } \\
\text { Four techniques: } \\
\text { Two conventional screws were installed in } \\
\text { each bone segment using a conventional } \\
\text { straight miniplate } \\
\text { Two locking screws were installed in each } \\
\text { bone segment using a locking straight } \\
\text { miniplate } \\
\text { Three conventional screws were installed in } \\
\text { the proximal segment and four conventional } \\
\text { screws were installed in the distal segment, } \\
\text { using a seven-hole conventional miniplate } \\
\text { Three locking screws were installed in the } \\
\text { proximal segment and four locking screws } \\
\text { were installed in the distal segment, using a } \\
\text { seven-hole locking miniplate }\end{array}$ & $\begin{array}{l}\text { Vertical loading at the second molar (side of } \\
\text { fixation) }\end{array}$ \\
\hline $\begin{array}{l}\text { Kalfarentzos et } \\
\text { al. } 2009\end{array}$ & $\begin{array}{l}\text { Synthetic } \\
\text { (replicas) }\end{array}$ & $\begin{array}{l}\text { Four techniques: } \\
\text { 3D miniplate }(2 \times 2 \text { holes, square, } 2 \mathrm{~mm}) \\
\text { 3D miniplate }(6 \times 2 \text { holes, curved, } 2 \mathrm{~mm}) \\
\text { Two miniplates (four holes, straight, } 2 \mathrm{~mm} \\
\text { and } 1.6 \mathrm{~mm}) \\
\text { One single miniplate (four holes, straight, } 2 \\
\mathrm{~mm})\end{array}$ & $\begin{array}{l}\text { Vertical loading at incisal and homolateral molar } \\
\text { region }\end{array}$ \\
\hline $\begin{array}{l}\text { Turgut et } \\
\text { al. } 2008\end{array}$ & Animal (sheep) & $\begin{array}{l}\text { Titanium 4-hole noncompression miniplates } \\
\text { and 11-hole reconstruction plates (Elektron } \\
\text { Medikal, Trimed, Turkey) } \\
\text { Six-millimeter monocortical screws, 13-mm } \\
\text { bicortical screws, and 13-mm reconstruction } \\
\text { screws } \\
\text { Four techniques: } \\
\text { Single plate upper border (Champy technique) } \\
\text { Biplanar dual-miniplate, upper border- } \\
\text { proximal } 3 \text { holes bicortical fourth hole } \\
\text { monocortical, lower border-bicortical } \\
\text { Biplanar, dual-miniplate, upper border- } \\
\text { monocortical, lower border-bicortical } \\
\text { Reconstruction plate }\end{array}$ & $\begin{array}{l}\text { Three-point bending test (Mandible body) } \\
\text { Compression test } \\
\text { (anterior and lateral mandible body) } \\
\text { Side-bending test } \\
\text { (mandible body) }\end{array}$ \\
\hline $\begin{array}{l}\text { Alkan et } \\
\text { al. } 2007\end{array}$ & Animal (sheep) & $\begin{array}{l}\text { Titanium 4-hole noncompression miniplates } \\
\text { (Electron Medical, Trimed, Turkey) and all } \\
\text { the screws were } 5 \mathrm{~mm} \text { in length, fabricated } \\
\text { titanium, and self-tapping } \\
\text { Four techniques: } \\
\text { Single plate upper border (Champy technique) } \\
\text { Biplanar plate placement (plates positioned in } \\
2 \text { planes) } \\
\text { Monoplanar plate placement (plates } \\
\text { positioned in } 1 \text { plane) } \\
\text { 3-dimensional curved angle strut plate } \\
\text { (Mondeal Medical Systems, Tuttlingen, } \\
\text { Germany) }\end{array}$ & $\begin{array}{l}\text { Vertical loading at mandibular angle } \\
\text { (side of fixation) }\end{array}$ \\
\hline $\begin{array}{l}\text { Haug et } \\
\text { al. } 2002\end{array}$ & $\begin{array}{l}\text { Synthetic } \\
\text { (polyurethane) }\end{array}$ & $\begin{array}{l}\text { All monocortical screws used were } 6.0 \mathrm{~mm} \text { in } \\
\text { length, fabricated of titanium, and self-tapping } \\
\text { and all bicortical screws were } 16.0 \mathrm{~mm} \text { in } \\
\text { length, fabricated of titanium, and self-tapping } \\
\text { Four techniques: These categories were } \\
\text { further subdivided into groups based on the } \\
\text { amount of offset. These groups were } 0.0-\mathrm{mm} \\
\text { offset, } 1.0 \text {-mm offset, and } 2.0-\mathrm{mm} \text { offset } \\
\text { Screws and plates were obtained from } \\
\text { Synthes Maxillofacial, (Paoli, PA) } \\
\text { Four techniques: }\end{array}$ & $\begin{array}{l}\text { Vertical loading at incisal edge and molar loading } \\
\text { (side of fixation) }\end{array}$ \\
\hline
\end{tabular}




\begin{tabular}{|c|c|c|c|}
\hline & & $\begin{array}{l}\text { Locking reconstruction plates secured at the } \\
\text { inferior border with } 2.4 \text {-mm outer thread } \\
\text { diameter bicortical locking screws } \\
\text { Nonlocking reconstruction plates secured at } \\
\text { the inferior border with } 2.4 \text {-mm outer thread } \\
\text { diameter bicortical nonlocking screws } \\
\text { Locking monocortical superior border plates } \\
\text { secured with } 2.0 \text {-mm outer thread diameter } \\
\text { locking screws } \\
\text { Nonlocking monocortical superior border } \\
\text { plates secured with } 2.0 \text {-mm outer thread } \\
\text { diameter nonlocking screws }\end{array}$ & \\
\hline $\begin{array}{l}\text { Haug et } \\
\text { al. } 2001\end{array}$ & $\begin{array}{l}\text { Synthetic } \\
\text { (polyurethane) }\end{array}$ & $\begin{array}{l}\text { A variety of techniques was evaluated as } \\
\text { follows: } \\
\text { Screw technique, monocortical superior } \\
\text { border plating techniques with varying sizes } \\
\text { of plates and screws, monocortical 2-plate } \\
\text { techniques with varying forms of fixation, } \\
\text { monocortical tension band systems with } \\
\text { associated bicortical stabilization plates of } \\
\text { various types, and various forms of } \\
\text { reconstruction plates }\end{array}$ & $\begin{array}{l}\text { Vertical loading at the incisal edge and then } \\
\text { repeated for contralateral loading in the molar } \\
\text { region }\end{array}$ \\
\hline $\begin{array}{l}\text { Wittenberg et } \\
\text { al. } 1997\end{array}$ & Animal (sheep) & $\begin{array}{l}\text { Three techniques: } \\
\text { Synthes reconstruction plate (S-recon) placed } \\
\text { near the inferior border. } \\
\text { Synthes mesh (S-mesh, Synthes, Paoli, PA, } \\
\text { USA) placed in the middle of the buccal } \\
\text { surface of the mandible } \\
\text { The Leibinger 3-D plate (L-3D, Leibinger- } \\
\text { Fischer, Irving, TX, USA) placed in the } \\
\text { middle of the buccal surface of the mandible }\end{array}$ & $\begin{array}{l}\text { Vertical load } 20 \mathrm{~mm} \text { anterior to the osteotomy } \\
\text { site (side of fixation) }\end{array}$ \\
\hline Haug et al. 1996 & $\begin{array}{l}\text { Synthetic } \\
\text { (polyurethane) }\end{array}$ & $\begin{array}{l}\text { Three techniques: } \\
\text { Four-hole titanium tension band } \\
\text { Self-tapping monocortical titanium screws } \\
(2.0 \mathrm{~mm} \text { outer thread diameter and } 4.0 \mathrm{~mm} \text { in } \\
\text { length) } \\
1.7 \mathrm{~mm} \text { thick, } 6.5 \mathrm{~mm} \text { wide, four-hole } \\
\text { titanium Limited Contact Dynamic } \\
\text { Compression Plate } \\
\text { Self-tapping bicortical titanium screws ( } 2.4 \\
\text { mm outer thread diameter and } 10.0 \mathrm{~mm} \text { in } \\
\text { length) } \\
\text { Thinner and smaller tension band at superior } \\
\text { border and thicker and larger stabilization } \\
\text { plate at inferior border } \\
\text { Thicker and larger stabilization plate at } \\
\text { superior border and thinner and smaller } \\
\text { tension band at inferior border } \\
\text { Two miniplate group used two thin plates: one } \\
\text { as stabilization plate and other as tension band } \\
\text { All plates were obtained from Synthes } \\
\text { Maxillofacial (Paoli, USA) }\end{array}$ & $\begin{array}{l}\text { Vertical load at the canine region (side of } \\
\text { fixation) }\end{array}$ \\
\hline $\begin{array}{l}\text { Shetty et } \\
\text { al. } 1995\end{array}$ & $\begin{array}{l}\text { Dentate } \\
\text { cadaveric } \\
\text { mandible }\end{array}$ & $\begin{array}{l}\text { Six techniques: } \\
\text { EDCP - lower border of the mandible with } \\
2.7 \text { mm non-self-tapping screws (Synthes } \\
\text { Maxillofacial, Paoli, PA) } \\
\text { Würzburg - Würzburg fracture plate applied } \\
\text { at the lower border of the mandible with 2.7- } \\
\text { mm non-self-tapping screws (Leibinger and } \\
\text { Fischer, Irving, TX) } \\
\text { Luhr - Titanium Luhr curved mandibular } \\
\text { compression system (MCS) plate applied at } \\
\text { the lower border with 2.7-ram self-tapping } \\
\text { screws (Howmedica Inc., Rutherford, NJ) }\end{array}$ & $\begin{array}{l}\text { Vertical load at the second molar, second } \\
\text { premolar (side of fixation) and first molar } \\
\text { (opposite side) }\end{array}$ \\
\hline
\end{tabular}




\begin{tabular}{|c|c|c|c|}
\hline & & $\begin{array}{l}\text { Lag Screw - Solitary lag screw (Synthes } \\
\text { Maxillofacial) applied across the fracture line } \\
\text { in a caudolateral cranio lingual fashion } \\
\text { Champy - Champy miniplate applied along } \\
\text { the broad vestibular area bounded by the } \\
\text { external oblique ridge with 2.0-mm } \\
\text { monocortical screws (W. Lorenz, } \\
\text { Jacksonville, FL) } \\
\text { Mennen - Mennen paraskeletal plate (CMW } \\
\text { Laboratories, Exeter, UK) }\end{array}$ & \\
\hline Choi et al. 1995 & $\begin{array}{l}\text { Cadaveric } \\
\text { mandible }\end{array}$ & $\begin{array}{l}\text { Two techniques: } \\
\text { 4-hole miniplate was carried out in the area of } \\
\text { the external oblique line as described by } \\
\text { Champy et al., } 1976 \\
\text { 4-hole miniplate was carried out in the area of } \\
\text { the external oblique line and a second 4-hole } \\
\text { plate using monocortical } 2.0 \mathrm{~mm} \text { screws was } \\
\text { applied at the inferior border of the mandible } \\
\text { All plates were obtained from Storz (USA) }\end{array}$ & $\begin{array}{l}\text { Vertical load at the } 47,37 \text { and } 41 / 31 \text { (central } \\
\text { groove of the } 47 \text { and } 37 \text {, interdental papilla in } \\
\text { case of the } 41 / 31 \text { ) }\end{array}$ \\
\hline $\begin{array}{l}\text { Shetty \& } \\
\text { Caputo. } 1992\end{array}$ & $\begin{array}{l}\text { Dentate } \\
\text { cadaveric } \\
\text { mandible } \\
\& \\
\text { composite } \\
\text { photoelastic } \\
\text { mandible } \\
\text { analog }\end{array}$ & $\begin{array}{l}\text { 2.7-mm A0 cortical screw (Synthes } \\
\text { Maxillofacial, Paoli, PA) of appropriate } \\
\text { length ( } 28 \text { to } 34 \mathrm{~mm}) \\
\text { Two techniques: } \\
\text { Interfragmentary displacement } \\
\text { Photoelastic analysis }\end{array}$ & $\begin{array}{l}\text { Vertical load at first premolar adjacent to the } \\
\text { fracture }\end{array}$ \\
\hline
\end{tabular}

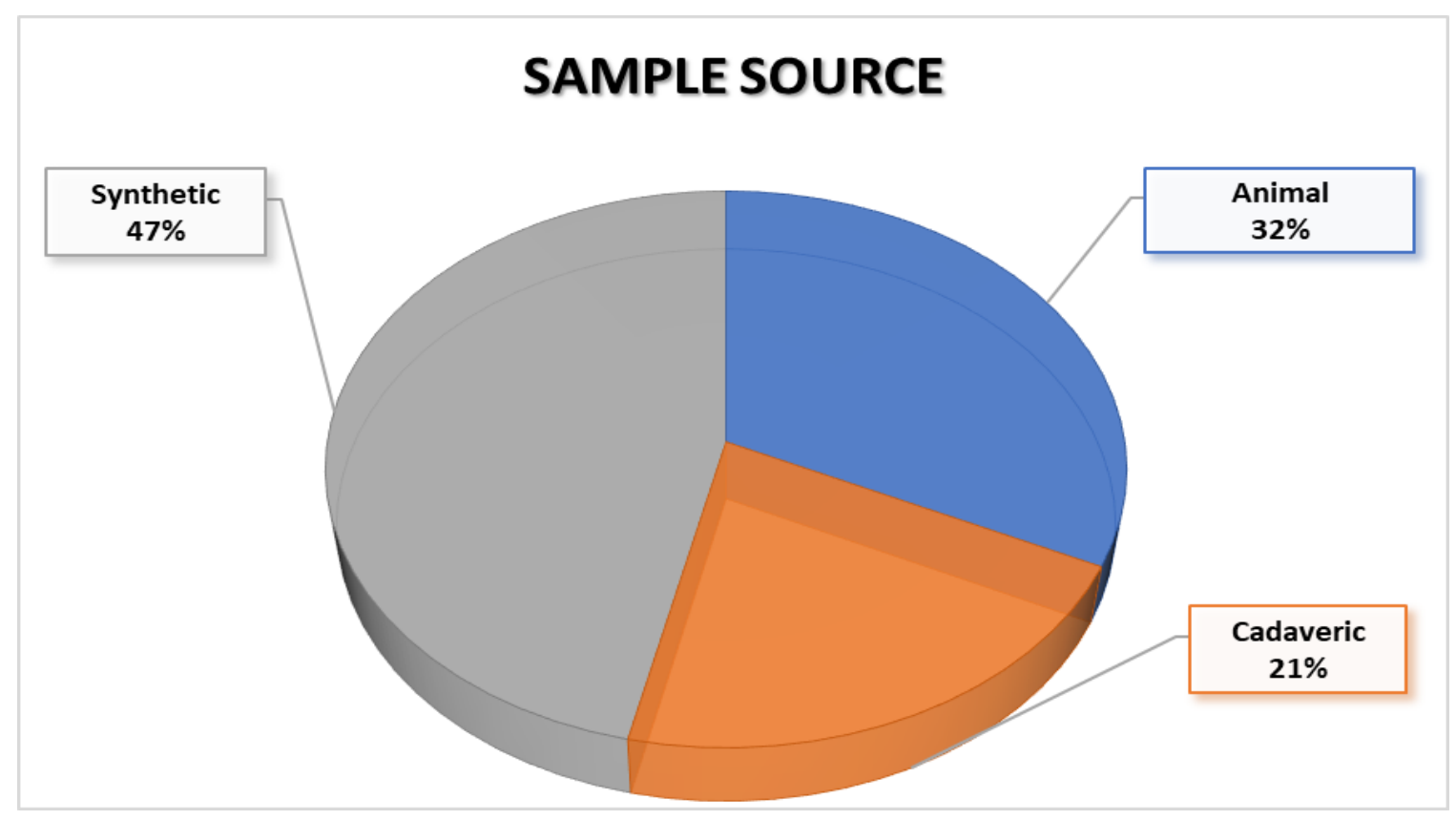

Figure 2: Distribution of the sample source across the included studies.

A variety of plates from different manufacturers was described as follows: titanium miniplates, resorbable miniplates (poly-DL-lactic acid; PDLLA), locking and non-locking systems, plates with tridimensional design, and reconstruction plates. The constitution of the plates was not adequately reported in most papers; however, some mentioned details regarding the materials.
A single-plating technique was the most performed technique in most articles, and the site for fixation was usually the external oblique ridge, according to principles of Champy et al. (1976), using a four-hole plate. However, a fixation on the intermediary portion of the fracture was also reported (middle of the buccal surface). The site of fixation if a second place was chosen was the inferior mandible border. However, if only the inferior border was plated, a reconstruction plate was used. Fixation only with screws was also reported (Shetty and Caputo 1992; Shetty et al., 
1995). One article reported fixation with a Herbert bone screw (Wallner et al., 2017). Table 2 provides a summary of the included biomechanical studies of mandibular angle fractures.

After the mandible was plated, most studies performed biomechanical tests applying loadings on the same side of the fixation, generally on the molar region. Other variations include loading on the canines, bicuspids, between the canine and first bicuspids, and $20 \mathrm{~mm}$ anterior to the osteotomy site. Some studies also applied loading the contralateral side and vertical loading at the central incisors. One article (Suer et al., 2014) reported using a machine stated to simulate the masticatory muscles, which was designed to apply vertical, lateral and tensile forces (Figure 3).

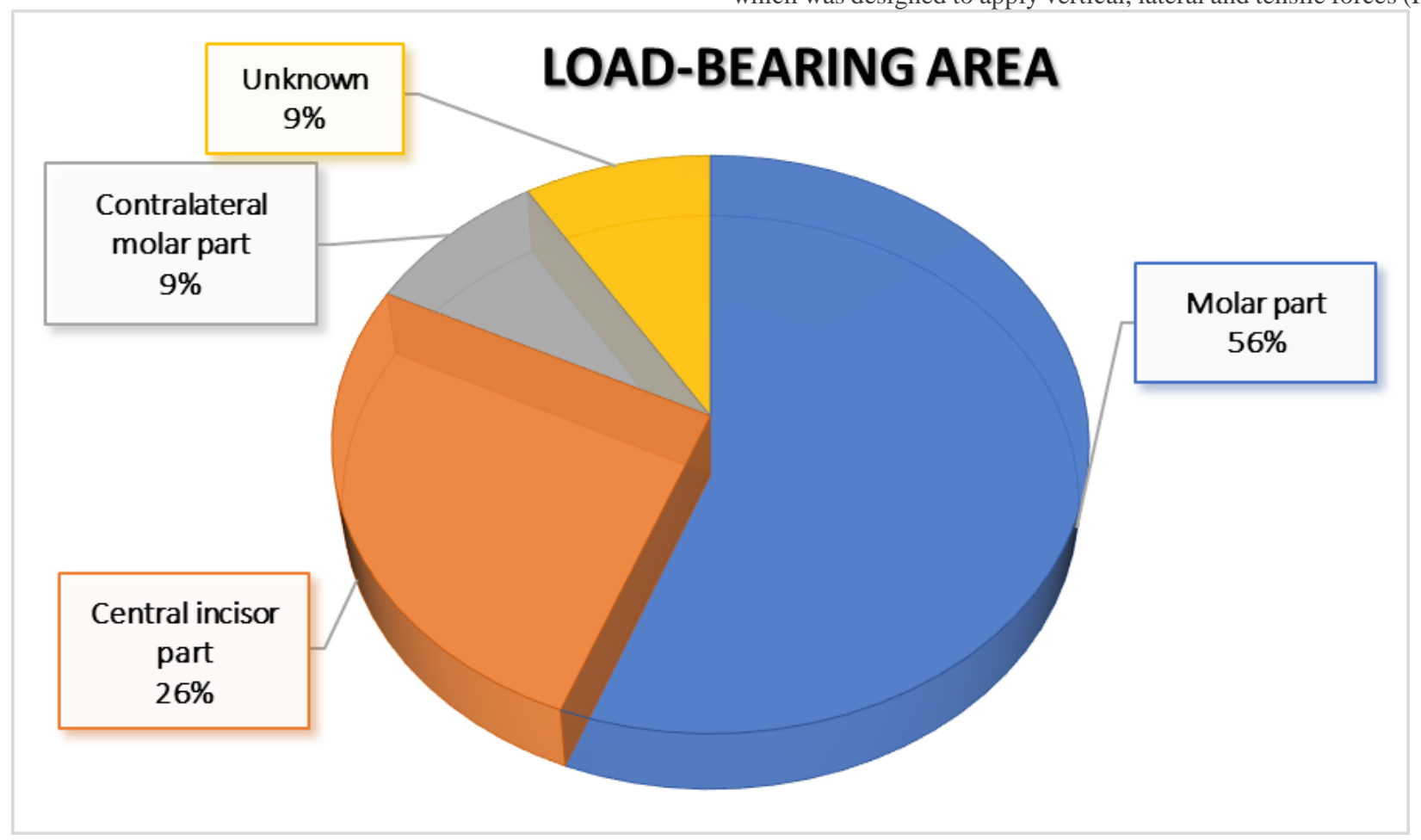

Figure 3: Distribution of the load-bearing area across the included studies.

\section{Discussion}

The aim of this study was to summarize the methodological aspects of biomechanical in vitro studies evaluating different plating and fixation techniques for mandibular angle fractures. Also, synthetic jawbone models, animal mandibles, and cadaver mandibles have been described in the scientific literature as the sample source in in vitro studies. They are used to evaluate the mechanical strength and stability of the different available fixation systems for the treatment of mandibular angle fractures (Wong et al., 2011; Guastaldi et al., 2014; Guastaldi et al., 2020). Due to the significant complications (i.e. infection, malocclusion) that may arise from the treatment of these fractures (Zimmermann et al., 2017), different treatment modalities have been proposed in the last 30 years aimed at improving fracture stability. These include using plates with different thickness (Alkan et al., 2007; Turgut et al., 2008), plates with different composition [i.e., commercially pure titanium versus titanium alloys (Guastaldi et al., 2014)], plates with three-dimensional configuration (Kalfarentzos et al., 2009; Oliveira et al., 2018), resorbable materials (Cural et al., 2018), lag screws (Shetty \& Caputo, 1992), and even bone adhesives (Cural et al., 2018).

The thin cross-section of the mandibular angle in addition sometimes to the presence of impacted third molars makes this region prone to fractures (Dhara et al., 2019), which may occur in association with multiple facial fractures and other patient morbidities (Le et al., 2020). Moreover, highenergy trauma (i.e. road traffic accidents) is usually associated with these injuries (Jaber et al., 2020) causing a comminution that furthers complicates treatment (Chen et al., 2018; Sikora et al., 2019). These factors make the treatment of mandibular angle fractures challenging and hinders carrying controlled studies to evaluate different treatments. Therefore, in vitro studies try to replicate patterns of mandibular angle fracture to overcome the limitations of standardized patient recruitment. Table 3 presents some potentialities and limitations of biomechanical studies.

It has been shown that the screw dimeter influences the distribution of stress/strains in holes, which is directly related to screw loosening (Yoon et al., 2014). Also, the results from computational modeling studies [i.e. finite element analysis (FEA)] simulating the distribution of stress/strains along fracture lines show that screw position is an additional factor that influences microstrains on holes (Guastaldi et al., 2020; Ramos et al., 2020). However, there are some limitations of computational models, such as the lack of mechanical testing to evaluate fatigue performance and the fact that FEA modelling is applied to a static situation. Therefore, in vitro studies help to validate the accuracy and precision of these models (Wong et al., 2011). Additionally, there are some testing machines that can simulate the action of the masticatory muscles (Suer et al., 2014), which is an important factor in the real clinical scenario. Also, incisal edge loading, along with molar loading, inflicts a combination of torsional and vertically deforming forces that are encountered clinically (Haug et al., 2002). Table 4 describe some information of available standards that should be used when performing biomechanical studies for mandibular angle fixation. 


\section{Potentialities}

Low cost when using polyurethane mandibles

When using polyurethane mandibles, standardization of the fracture, plating technique(s)

Objective assessments through biomechanical testing

Easy setting of an experimental design model

\section{Limitations}

Higher cost, if using cadaveric mandibles from larger animals (i.e. swine, sheep)

Difficulty in reproducing the true clinical scenario (fracture patterns are more complex)

Difficult to reproduce the action of complex masticatory forces

Differences in results are likely to occur depending on the research model:

-Polyurethane cannot distinguish cortical bone and cancellous bone -With animal bones, it is difficult to standardize the size and bone density

Table 3: Potentialities and limitations of biomechanical studies

\begin{tabular}{|c|c|c|}
\hline Standard \# & Standard description & Website \\
\hline $\begin{array}{l}\text { ASTM: } \\
\text { F543-17 }\end{array}$ & $\begin{array}{l}\text { Standard Specification and Test Methods for Metallic Medical } \\
\text { Bone Screws }\end{array}$ & https://www.astm.org/Standards/F543.htm \\
\hline $\begin{array}{l}\text { ASTM: } \\
\text { F1839-08(2016) }\end{array}$ & $\begin{array}{l}\text { Standard Specification for Rigid Polyurethane Foam for Use as a } \\
\text { Standard Material for Testing Orthopaedic Devices and } \\
\text { Instruments }\end{array}$ & https://www.astm.org/Standards/F1839.htm \\
\hline
\end{tabular}

Table 4: Information of available standards that should be used when performing biomechanical studies for mandibular angle fixation.

Mandibular angle fractures still represent one of the most controversial issues in the relevant literature, especially regarding the recommended treatment. The evolution of internal fixation was aided by the discovery of biocompatible materials that resisted corrosion, such as vitallium and titanium. Currently, titanium is the metal of choice for fixation plates, mainly because of its high biocompatibility, ease of manipulation, and the potential for no second surgery. Additionally, efforts have been made to increase the use of resorbable plates. However, few studies have investigated the biomechanical behavior of the stability when using resorbable plates and screws (Cural et al., 2018). Although a spectrum of techniques for treatment of angle fractures with plates/screws has been proposed in the literature (Table 5), and what constitutes adequate stability at a fractured mandibular angle remains controversial (Levy et al., 1991; Schierle et al., 1997; Ellis, 1999).

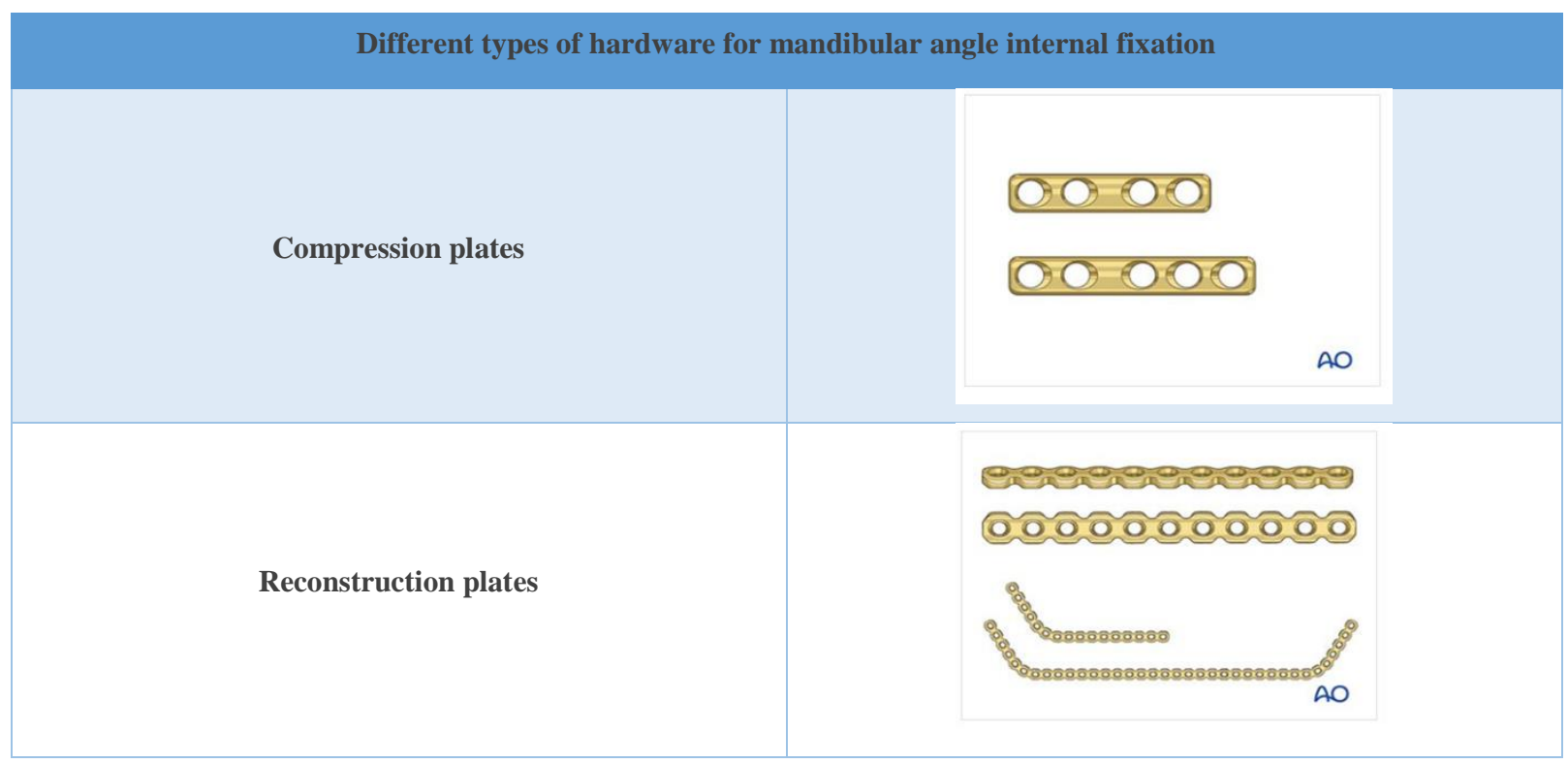




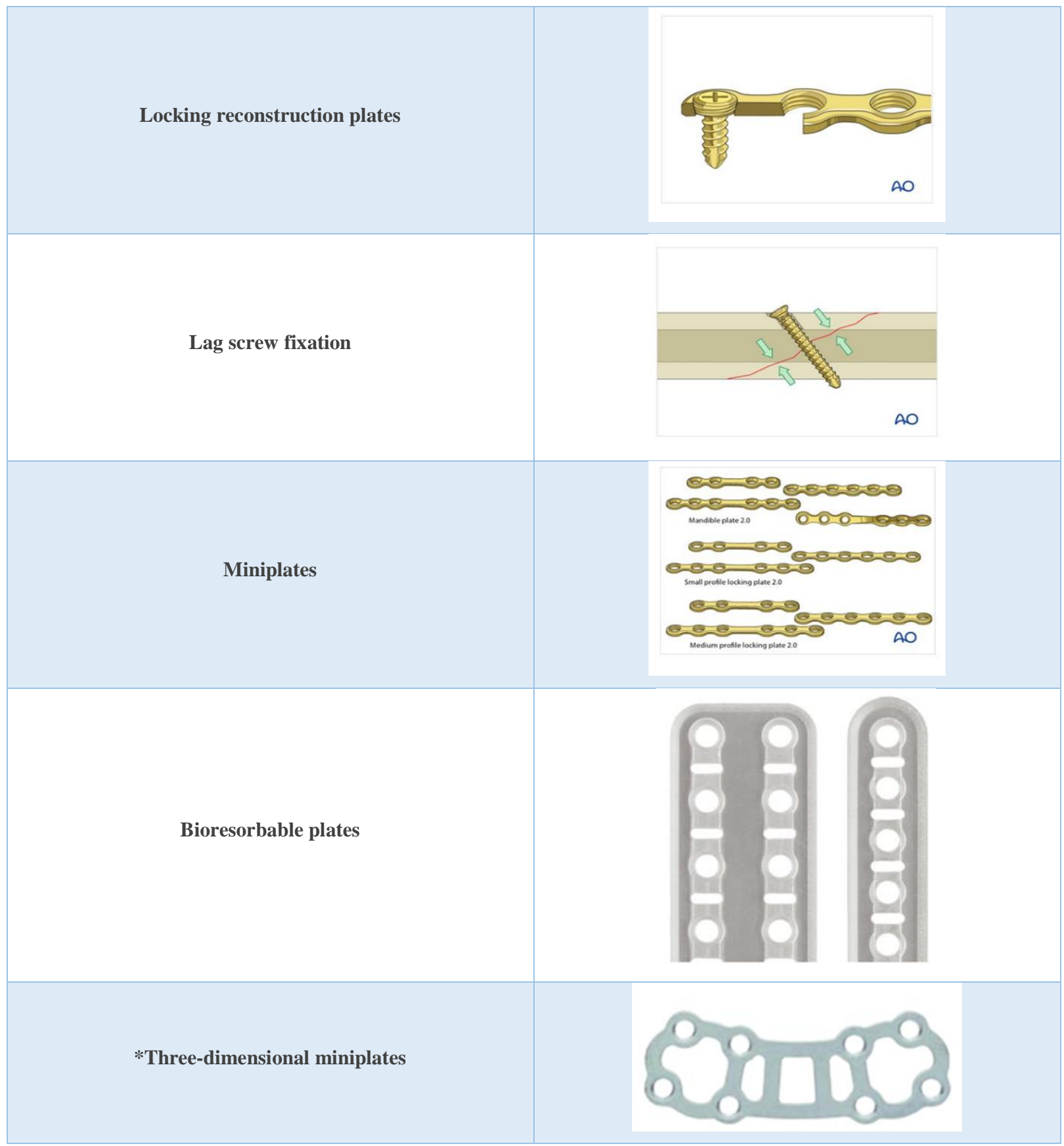

Table 5: Summary of different hardware for mandibular angle fractures

\section{Conclusion}

Biomechanical studies of mandibular angle fracture fixation are challenging due to the complex forces acting on the mandible. They represent a valuable methodology to identify trends in behavior, however these trends need to be verified with clinical investigations. There isn't a consensus regarding the "gold standard" treatment of mandibular angle fractures. However biomechanical studies contribute to the assessment of the biomechanical performance for the stable internal fixation of mandibular angle fractures. Also, they are important to develop new techniques and help to answer questions on fatigue performance and fracture strength on the gross level.

\section{Conflict of interest}

The authors declare that they have no competing interests.

\section{References}

1. Afrooz PN, Bykowski MR, James IB, Daniali LN, ClavijoAlvarez JA. (2015). The Epidemiology of Mandibular Fractures in the United States, Part 1: A Review of 13,142 Cases from the US National Trauma Data Bank. Journal of Oral Maxillofacial Surgery. 73 (12): 2361-2366.

2. Alkan, Alper, Nükhet Çelebi, Bora Özden, Burcu Baş, and Samet İnal. (2007). "Biomechanical Comparison of Different Plating Techniques in Repair of Mandibular Angle Fractures." Oral Surgery, Oral Medicine, Oral Pathology, Oral Radiology, and Endodontology. 104 (6): 752-756. 
3. Allareddy, Veerasathpurush, Veerajalandhar Allareddy, and Romesh P. Nalliah. (2011). "Epidemiology of Facial Fracture Injuries." Journal of Oral and Maxillofacial Surgery. 69 (10): 2613-2618.

4. Ben Said, Muhammed, Senem Yildirimturk, and Yigit Sirin. (2019). "The Effect of the Mandibular Plane Angle on Fracture Line Stability: An Ex Vivo Experimental Study." Dental Traumatology. 35 (3): 181-187.

5. Bormann, Kai-Hendrik, Sarah Wild, Nils-Claudius Gellrich, Horst Kokemüller, Constantin Stühmer, Rainer Schmelzeisen, and Ralf Schön. (2009). "Five-Year Retrospective Study of Mandibular Fractures in Freiburg, Germany. Incidence, Etiology, Treatment, and Complications." Journal of Oral and Maxillofacial Surgery. 67 (6): 1251-1255

6. Cankaya, Abdulkadir Burak, Metin Berk Kasapoglu, Mehmet Ali Erdem, and Cetin Kasapoglu. (2018). "Effects of Polymethylmethacrylate on the Stability of Screw Fixation in Mandibular Angle Fractures: A Study on Sheep Mandibles." International Journal of Medical Sciences. 15 (13): 1466-1471.

7. Champy M, Lodde JP, Jaeger JH, Wilk A. (1976). "Biomechanical basis of mandibular osteosynthesis according to the F.X. Michelet method." Revue de Stomatologie, de Chirurgie Maxillo-faciale et de Chirurgie Orale. 77 (1): 248-251.

8. Choi BH, Yoo JH, Kim KN, Kang HS. (1995). "Stability Testing of a Two Miniplate Fixation Technique for Mandibular Angle Fractures. An in Vitro Study." Journal of Cranio-Maxillofacial Surgery. 23 (2): 122-125.

9. Cural, Ülkem, Berkem Atalay, and Mustafa Sami Yildirim. (2018). "Comparison of Mechanical Stabilization of the Mandibular Angulus Fracture Fixation, With Titanium Plates and Screws, Resorbable Plates and Screws, and Bone Adhesives." Journal of Craniofacial Surgery. 29 (7): 1780-1787.

10. Ellis E, Sinn DP. (1993). "Treatment of Mandibular Angle Fractures Using Two 2.4-Mm Dynamic Compression Plates." Journal of Oral and Maxillofacial Surgery. 51 (9): 969-973.

11. Ellis E. (1993). "Treatment of Mandibular Angle Fractures Using the AO Reconstruction Plate." Journal of Oral and Maxillofacial Surgery. 51 (3): 250-254.

12. Ellis E. (1999). "Treatment Methods for Fractures of the Mandibular Angle." International Journal of Oral and Maxillofacial Surgery. 28 (4): 243-52.

13. Erdmann, Detlev, Keith E. Follmar, Marlieke DeBruijn, Anthony D. Bruno, Sin-Ho Jung, David Edelman, Srinivasan Mukundan, and Jeffrey R. Marcus. (2008). "A Retrospective Analysis of Facial Fracture Etiologies." Annals of Plastic Surgery. 60 (4): 398-403.

14. Gabrielli, Marisa Aparecida Cabrini, Mário Francisco Real Gabrielli, Elcio Marcantonio, and Eduardo Hochuli-Vieira. (2003). "Fixation of Mandibular Fractures with 2.0-Mm Miniplates: Review of 191 Cases." Journal of Oral and Maxillofacial Surgery. 61 (4): 430-436.

15. Gassner, Robert, Tarkan Tuli, Oliver Hächl, Ansgar Rudisch, and Hanno Ulmer. (2003). "Cranio-Maxillofacial Trauma: A 10 Year Review of 9543 Cases with 21067 Injuries." Journal of CranioMaxillofacial Surgery. 31 (1): 51-61

16. Guastaldi FPS, Hochuli-Vieira E, Guastaldi AC. (2014). "Biomechanical Study in Polyurethane Mandibles of Different Metal Plates and Internal Fixation Techniques, Employed in Mandibular Angle Fractures.” Journal of Craniofacial Surgery 25 (6): 2246-2250.

17. Guastaldi FPS, Martini AP, Rocha EP, Hochuli-Vieira E, Guastaldi AC. (2020). "Ti-15Mo Alloy Decreases the Stress Concentration in Mandibular Angle Fracture Internal Fixation
Hardware." Journal of Maxillofacial and Oral Surgery. 19: 314 320.

18. Haug RH, Street CC, Goltz M. (2002). "Does Plate Adaptation Affect Stability? A Biomechanical Comparison of Locking and Nonlocking Plates.” Journal of Oral and Maxillofacial Surgery. 60 (11): 1319-1326.

19. Haug RH, Barber JE, Reifeis R. (1996). "A Comparison of Mandibular Angle Fracture Plating Techniques.” Oral Surgery, Oral Medicine, Oral Pathology, Oral Radiology, and Endodontology. 82 (3): 257-263.

20. Haug, Richard H., Tirbod T. Fattahi, and Michele Goltz. (2001). "A Biomechanical Evaluation of Mandibular Angle Fracture Plating Techniques." Journal of Oral and Maxillofacial Surgery. 59 (10): 1199-1210.

21. Iizuka, Tateyuki, Christian Lindqvist, Dorrit Hallikainen, and Pertti Paukku. (1991). "Infection after Rigid Internal Fixation of Mandibular Fractures: A Clinical and Radiologic Study.” Journal of Oral and Maxillofacial Surgery. 49 (6): 585-593.

22. Jafarian, M., F. Pourdanesh, M. Esmaeelinejad, N. Dehghani, F. Aghdashi, and R. Tabrizi. (2015). "Assessment of Compression and Strength of Divergent Screws Mounted on Miniplates for Fixation of Mandibular Fractures: An in Vitro Experimental Study." British Journal of Oral and Maxillofacial Surgery. 53 (7): 613-617.

23. Jain, Manoj Kumar, and Mohan Alexander. (2009). "The Need of Postoperative Radiographs in Maxillofacial Fractures - A Prospective Multicentric Study." British Journal of Oral and Maxillofacial Surgery. 47 (7): 525-529.

24. Kalfarentzos, Evagelos F., Despoina Deligianni, Georgios Mitros, and Minos Tyllianakis. (2009). "Biomechanical Evaluation of Plating Techniques for Fixing Mandibular Angle Fractures: The Introduction of a New 3D Plate Approach." Oral and Maxillofacial Surgery. 13 (3): 139-144.

25. Levy, Frederic E., Robert W. Smith, Rick M. Odland, and Lawrence J. Marentette. (1991). "Monocortical Miniplate Fixation of Mandibular Angle Fractures." Archives of Otolaryngology-Head \& Neck Surgery. 117 (2): 149-154.

26. Lieger, Olivier, Benoit Schaller, Alexander Bürki, and Philippe Büchler. (2015). "Biomechanical Evaluation of Different AngleStable Locking Plate Systems for Mandibular Surgery.” Journal of Cranio-Maxillofacial Surgery. 43 (8): 1589-1594.

27. Medeiros, Raquel Correia de, Eder Alberto Sigua, Pablo Navarro, Sergio Olate, and Jose Ricardo Albergaria Barbosa. (2016). "In Vitro Mechanical Analysis of Different Techniques of Internal Fixation of Combined Mandibular Angle and Body Fractures." Journal of Oral and Maxillofacial Surgery. 74 (4): 778-785.

28. Muñante-Cardenas, JoseLuis, and LuisAugusto Passeri. (2015). "Biomechanical Comparison of Four Mandibular Angle Fracture Fixation Techniques." Craniomaxillofacial Trauma \& Reconstruction 8 (2): 123-128.

29. Negreiros Lyrio, Mariana C., Marcelo Silva Monnazzi, Marcio De Moraes, Eduardo Hochuli-Vieira, José Mauricio Nunes Reis, and Valfrido Antonio Pereira-Filho. (2014). "Comparison of Compressive Strength between Three Different Plates for Mandibular Angle Fractures Fixation." Journal of CranioMaxillofacial Surgery. 42 (5): 277-280.

30. Pektas ZO, Bayram B, Balcik C, Develi T, Uckan S. (2012). "Effects of Different Mandibular Fracture Patterns on the Stability of Miniplate Screw Fixation in Angle Mandibular Fractures." International Journal of Oral and Maxillofacial Surgery. 41 (3): 339-343.

31. Pereira-Filho, Valfrido Antonio, Luis Fernando de Gorla Oliveira, José Maurício dos Santos Nunes Reis, Marisa Aparecida Cabrini Gabrielli, Rubens Spin Neto, and Marcelo Silva Monnazzi. 
(2016). "Evaluation of Three Different Osteosynthesis Methods for Mandibular Angle Fractures: Vertical Load Test.” Journal of Craniofacial Surgery. 27 (7): 1770-1773.

32. Ribeiro-Junior, P. D., O. Magro-Filho, K. A. Shastri, and M. B. Papageorge. (2010). "In Vitro Evaluation of Conventional and Locking Miniplate/Screw Systems for the Treatment of Mandibular Angle Fractures." International Journal of Oral and Maxillofacial Surgery. 39 (11): 1109-1114.

33. Schierle HP, Schmelzeisen R, Rahn B, Pytlik C. (1997). "One- or Two-Plate Fixation of Mandibular Angle Fractures?" Journal of Cranio-Maxillofacial Surgery. 25 (3): 162-168.

34. Schmelzeisen, Rainer, Terry McIff, and Berton Rahn. (1992). "Further Development of Titanium Miniplate Fixation for Mandibular Fractures. Experience Gained and Questions Raised from a Prospective Clinical Pilot Study with 2.0 Mm Fixation Plates.” Journal of Cranio-Maxillofacial Surgery. 20 (6): 251-256.

35. Schug, Thomas, Herbert Rodemer, Walter Neupert, and Josef Dumbach. (2000). "Treatment of Complex Mandibular Fractures Using Titanium Mesh.” Journal of Cranio-Maxillofacial Surgery 28 (4): 235-237.

36. Shetty V, Caputo A. (1992). "Biomechanical Validation of the Solitary Lag Screw Technique for Reducing Mandibular Angle Fractures.” Journal of Oral and Maxillofacial Surgery. 50 (6): 603 607.

37. Shetty V, McBrearty D, Fourney M, Caputo A. (1995). "Fracture Line Stability as a Function of the Internal Fixation System: An in Vitro Comparison Using a Mandibular Angle Fracture Model.” Journal of Oral and Maxillofacial Surgery. 53 (7): 791-801.

38. Sittitavornwong, Somsak, Douglas Denson, David Ashley, David Cruz Walma, Sarah Potter, and Jonathan Freind. (2018). "Integrity of a Single Superior Border Plate Repair in Mandibular Angle Fracture: A Novel Cadaveric Human Mandible Model.” Journal of Oral and Maxillofacial Surgery 76 (12): 26111-26118.

39. Suer BT, Kocyigit ID, Kaman S, Tuz HH, Tekin U, Atil F. (2014). "Biomechanical Evaluation of a New Design Titanium Miniplate for the Treatment of Mandibular Angle Fractures." International Journal of Oral and Maxillofacial Surgery. 43 (7): 841-845.

40. Trivellato PFB, Pepato AO, Ribeiro MC, Sverzut CE, Trivellato AE. (2014). "In Vitro Evaluation of the Resistance of a 2.0-Mm Titanium Fixation System in the Sectioned Angle without Continuity of the Inferior Border of the Mandible." International Journal of Oral and Maxillofacial Surgery. 43 (5): 559-563.

41. Turgut, Gürsel, Mahmut Ulvi Kayah, Ali Tufan Soydan, Oguzhan Gündüz, Serdar Salman, Faik Oktar, and Lütfü Bas. (2008). "Biomechanical Comparison of a New Technique of Mandibular Angle Fractures: Biplanar and Bicortical Superior Proximal 3 Holes and Bicortical Inferior Plate Fixation." Journal of Craniofacial Surgery. 19 (2): 428-432.

42. Wallner, Jürgen, Knut Reinbacher, Matthias Feichtinger, Mauro Pau, Georg Feigl, Franz Quehenberger, Katja SchwenzerZimmerer, and Wolfgang Zemann. (2017). "Osteosynthesis Using Cannulated Headless Herbert Screws in Mandibular Angle Fracture Treatment: A New Approach?" Journal of CranioMaxillofacial Surgery 45 (4): 526-539

43. Wittenberg, Joerg M., Debl P. Mukherjee, Brian R. Smith, and Robert N. Kruse. (1997). "Biomechanical Evaluation of New Fixation Devices for Mandibular Angle Fractures." International Journal of Oral and Maxillofacial Surgery 26 (1): 68-73.

44. Zachariades, Nicholas, Michael Mezitis, Constintine Mourouzis, Demetrius Papadakis, and Athena Spanou. (2006). "Fractures of the Mandibular Condyle: A Review of 466 Cases. Literature Review, Reflections on Treatment and Proposals." Journal of Cranio-Maxillofacial Surgery 34 (7): 421-432.

45. Zimmermann, Catarina, Anders Henningsen, Kai-Olaf Henkel, Jan Klatt, Christian Jürgens, Klaus Seide, and Benjamin Kienast. (2017). "Biomechanical Comparison of a Multidirectional Locking Plate and Conventional Plates for the Osteosynthesis of Mandibular Angle Fractures-A Preliminary Study." Journal of Cranio-Maxillofacial Surgery. 45 (12): 1913-1920.

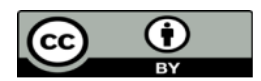

This work is licensed under Creative Commons Attribution 4.0 License

To Submit Your Article Click Here: Submit Manuscript

DOI: $10.31579 / 2643-6612 / 020$
Ready to submit your research? Choose Auctores and benefit from:

$>$ fast, convenient online submission

$>$ rigorous peer review by experienced research in your field

$>$ rapid publication on acceptance

$>$ authors retain copyrights

$>$ unique DOI for all articles

$>$ immediate, unrestricted online access

At Auctores, research is always in progress.

Learn more auctoresonline.org/journals/dentistry-and-oral-maxillofacialsurgery 